\title{
Impacto econômico da doença de Alzheimer no Brasil: é possível melhorar a assistência e reduzir custos?
}

\author{
Economic impact of Alzheimer's Disease in Brazil: \\ is it possible to improve care and minimize costs?
}

\author{
Beatriz Aparecida Ozello Gutierrez ${ }^{1}$ \\ Henrique Salmazo da Silva ${ }^{1}$ \\ Cristina Guimarães ${ }^{2}$ \\ Antonio Carlos Campino ${ }^{2}$
}

\footnotetext{
${ }^{1}$ Escola de Artes, Ciências e Humanidades, Universidade de São Paulo. Av. Arlindo Bétio 1000, Ermelino Matarazzo. 03828-000 São Paulo SP Brasil. biagutierrez@usp.br ${ }^{2}$ Faculdade de Economia, Administração e Contabilidade, Universidade de São Paulo.
}

\begin{abstract}
The scope of this study is to discuss data relating to the costs involved with Alzheimer's Disease and the initiatives in terms of care in order to reduce costs and help family members and caregivers to cope with the disease. The biggest cost for people with mild Alzheimer's Disease is the cost of time spent by the unremunerated caregiver, and for those at the advanced stages of the disease it is the cost of institutional care. In this respect, the literature proposes the adoption of models of care that maximize the functional independence of the elderly and maintain their skills, such as Day Care Centers for the Elderly and rehabilitation programs and support offered to the elderly and their family members. These models of care need to be discussed, structured and deployed in the context of the Brazilian reality.
\end{abstract}

Key words Elderly, Cost, Alzheimer's disease, Dementia
Resumo O objetivo deste estudo foi discutir dados relativos aos custos da Doença de Alzheimer (DA) e as iniciativas assistenciais para reduzir custos e auxiliar a família e os cuidadores no manejo com a doença. O fator de maior custo para as pessoas com DA leve foi o do tempo do cuidador não remunerado, enquanto que para aqueles com a doença em estágio avançado foi o dos cuidados relativos à institucionalização. Frente a esse panorama a literatura propõe a adoção de modelos de atenção que maximizem a independência funcional do idoso e a manutenção de suas habilidades, como a implantação do Centro Dia para Idosos e de programas de reabilitação e amparo ao idoso e família. Esses modelos de atenção precisam ser discutidos, estruturados e implantados na realidade brasileira.

Palavras-chave Idoso, Custo, Doença de Alzheimer, Demência 


\section{Introdução}

O envelhecimento acompanhado pelas síndromes demenciais é uma realidade que tende a aumentar nos próximos anos e em especial na velhice avançada.

Projeções de prevalência e incidência indicam que ocorrerá um crescimento mais elevado do número de pessoas com demência em países em desenvolvimento, que estão em transição demográfica. O número total de pessoas que sofrem de demência mundial em 2010 foi estimado em 35,6 milhões e é previsto que este número quase dobre a cada 20 anos - para 65,7 milhões em 2030 e 115,4 milhões em 2050. O número total de novos casos de demência a cada ano, no mundo, é de quase 7,7 milhões, o que implica um novo caso a cada quatro segundos ${ }^{1}$.

Dentre as demências destaca-se a Doença de Alzheimer (DA), que afeta 25 milhões de pessoas em todo o mundo ${ }^{2}$. Nos EUA, a prevalência da DA foi estimada em 4,5 milhões em 2000, e projetada para 13,2 milhões (um aumento de quase três vezes) em $2050^{3}$. A DA constitui aproximadamente $70 \%$ de todos os casos de demência ${ }^{4,5}$, sendo que a incidência aumenta com a idade, dobrando a cada cinco/ dez anos. Para as pessoas entre as idades de 6569, 70-74, 75-79, 80-84 e 85, a incidência da DA tem sido estimada em $0,6 \%, 1,0 \% ; 2,0 \%, 3,3 \%$ e $8,4 \%{ }^{6}$, respectivamente. A prevalência também aumenta exponencialmente com a idade, passando de 3\% entre os 65-74 anos, para quase 50\% entre os mais de 85 anos $^{6}$.

No Brasil, projeções indicam que a prevalência média apresenta-se mais alta que a mundial. Na população com 65 anos e mais, passando de $7,6 \%$ para $7,9 \%$ entre 2010 e 2020 , ou seja, 55.000 novos casos por ano ${ }^{7}$.

Partindo da premissa que a DA é uma doença crônica e neurodegenerativa que aumenta significativamente os custos dos cuidados de saúde e afeta a vida dos pacientes acometidos e seus cuidadores, é de suma importância pensar na reorganização dos serviços e na redução dos gastos associados à assistência, auxiliando os gestores, a execução de políticas públicas e os cuidados organizados pelas famílias. Dessa maneira, o objetivo deste estudo foi apresentar, por meio de revisão não sistemática da literatura, os dados relativos aos gastos da DA e as iniciativas assistenciais que poderiam ser mobilizadas a fim de reduzir gastos e auxiliar a família e os cuidadores a manejarem a doença, e discutir o impacto econômico da DA no Brasil, propondo estratégias assistenciais e re- dução de gastos relativos aos cuidados de longa duração.

\section{Método}

A busca de artigos nacionais e internacionais ocorreu na base de dados Scopus, Medline e SciELO a partir de 1998 até 2012. As palavraschave utilizadas foram: demência, doença de Alzheimer, prevalência, economia, custo e cuidadores, sendo todos os termos em combinação com a doença de Alzheimer e demência nos idiomas inglês, espanhol e português. Foram selecionados 82 resumos a partir do título. No entanto, 23 artigos foram lidos na íntegra, pois atendiam os objetivos desse estudo. Também se incluiu alguns trabalhos considerados relevantes para alicerçar o conteúdo pretendido.

\section{Impacto econômico \\ da Doença de Alzheimer}

A elevada prevalência de DA nas idades mais avançadas é produto das melhorias no tratamento e no uso de tecnologias que podem aumentar os anos de vida, mesmo na velhice acompanhada por enfermidades. Embora a progressão da demência e dos sintomas associados possa ser minimizada, os custos e as implicações do aumento da projeção delas estão presentes nas famílias e nos serviços de saúde.

Os custos dos cuidados com demência estão aumentando rapidamente nos países de baixo e médio rendimento ${ }^{8}$. As razões que foram atribuídas ao aumento dos custos são: rápido aumento do número de pessoas com demência nessas regiões; aumento substancial dos salários médios, usado para calcular o custo de oportunidade ou custo de substituição do cuidado informal; custos muito elevados com os cuidados de saúde pagos pela população de alguns países de rendimento médio ${ }^{8}$.

Sabe-se que o gasto total mundial na sociedade, empregado por causa da demência, com base em uma população de 34,4 milhões de pessoas com este mal, foi estimado em aproximadamente U\$ 422 milhões, em 2009, incluindo U\$ 142 milhões para cuidados informais $(34 \%)^{8}$. No entanto, a ausência de informação sobre os custos diretos com a DA e outras demências impossibilita a avaliação dos custos em países de baixo e médio rendimento ${ }^{8}$.

Bloom et al. ${ }^{9}$, ao observarem que o custo da DA é elevado, advertiram que as estimativas 
confiáveis não estavam disponíveis. Acreditam que os custos são suscetíveis às mudanças demográficas esperadas em todos os países e que essas estimativas de custos variam bastante afetando o custo real da DA e sua aplicabilidade aos serviços assistenciais.

Em 1998, Meek et al. ${ }^{10}$ afirmaram que em termos de custos totais para a sociedade, a DA era a terceira doença mais cara nos EUA, vindo após o câncer e as doenças coronarianas. E ainda, Brookmeyer et al. ${ }^{11}$ citaram que o impacto dos custos advindos do tratamento relacionados aos cuidados de saúde devido à DA seria enorme, trazendo uma marca para a economia dos EUA de até U\$ 36 bilhões por ano. Estimativas recentes revelaram que os custos associados à demência no Reino Unido são de 17 bilhões de euros por ano, custo que está estimado em 50 bilhões de euros para 2038, com incidência de 1,4 milhões de novos casos por ano ${ }^{12}$. O custo social dos cuidados da DA aumenta drasticamente com o aumento da severidade da doença, sendo a institucionalização o principal motivo ${ }^{13}$.

Para Schneider et al. ${ }^{14}$ e Cuijpers ${ }^{15}$ a ausência de tratamento dos sintomas comportamentais e psicológicos dos pacientes com DA estava associada à síndrome burnout do cuidador, ocasionando ônus, estresse, cansaço mental e depressão em quem cuida; e acarretando a institucionalização desses pacientes, a insuficiente gestão de suas comorbidades e os custos elevados dos cuidados de saúde.

Apesar de a literatura internacional enfatizar a relação DA x custos, no Brasil, os pesquisadores ainda estão atrelados apenas aos dados epidemiológicos da $\mathrm{DA}^{16-18}$, visto que é incipiente na literatura científica trabalhos que identificarem pesquisas abordando custos com os pacientes brasileiros com $\mathrm{DA}^{19}$.

Veras et al. ${ }^{19}$, ao investigarem 41 famílias de portadores de demência residentes no município do Rio de Janeiro, constataram que a projeção de custos associados aos cuidados de idosos com este mal atingia cerca de dois terços da renda familiar, encontrando-se um amento de 75\% quando os idosos estavam nos estágios iniciais da doença e $80 \%$ quando outras doenças crônicas foram levadas em consideração.

Embora a família represente a base principal dos cuidados e da atenção ao idoso com demência, é preciso considerar que, muitas vezes, os cuidadores familiares, em $90 \%$ dos casos filhas e esposas dos pacientes, veem-se sobrecarregados e sem o suporte necessário para promover os cuidados, ações que poderiam ser auxiliadas por mobilizações governamentais e acompanhadas por serviços formais de atenção ${ }^{20}$.

Por outro lado, considerando que nas últimas décadas houve redução do número de filhos e a inserção da mulher no mercado de trabalho, existe uma parcela considerável de idosos com declínio cognitivo que moram sozinhos, chegando a $22,6 \%$ do total dos que participaram do projeto $\mathrm{SABE}^{21}$. Entre esses idosos que moravam sozinhos, $65,1 \%$ referiram que não recebiam auxílio para realizar as Atividades Básicas de Vida Diária (ABVD), tais como locomover-se, ir ao banheiro, alimentar-se, entre outras. Esses resultados nos alertam quanto à provável dificuldade que esses indivíduos desamparados têm no seu dia a dia para conseguir manter sua capacidade funcional preservada ou mesmo sobreviver com suas incapacidades tentando manter uma qualidade de vida digna.

As questões da capacidade funcional e autonomia do idoso podem ser mais importantes que a própria questão da morbidade, pois se relacionam diretamente à qualidade de vida. Pesquisa realizada na região metropolitana de Belo Horizonte, Brasil, mostrou que a prevalência de incapacidades entre idosos foi alta e que as condições crônicas associadas à incapacidade na população estudada são passíveis de prevenção ${ }^{22}$.

Análise de inquérito domiciliar realizado entre pessoas com idade igual ou superior a 60 anos identificou déficit crescente de acordo com o aumento da idade em três indicadores de capacidade funcional: alimentar-se, tomar banho ou ir ao banheiro; abaixar-se, ajoelhar-se ou curvar-se; caminhar cerca de um quilômetro ${ }^{23}$.

Nesse sentido, o custo financeiro devido à presença de 800.000 pessoas vivendo com demência no Reino Unido foi superior a 23 bilhões de euros em 2012, em média de 29.746 euros por pessoa ${ }^{24}$. Essa população com demência, no Reino Unido, conta com 670 mil cuidadores. Sob esse aspecto, a análise dos resultados encontrados em estudo longitudinal referente à ocorrência de custos com a prestação de cuidados a pacientes com DA, realizado por Zhu et al. ${ }^{25}$, nos Estados Unidos da América, sugeriu que a dependência dos pacientes fornece uma contribuição significativa na explicação das variações nos custos da saúde na presença de DA e que as pequenas mudanças na dependência desses pacientes estão associadas a grandes mudanças nos custos de atendimento. Assim, as intervenções que melhorem ou preservem a independência ou, ainda, atrasem e retardem o declínio funcional desses pacientes podem estar ligadas à redução de custos. 


\section{Otimização dos serviços, redução de custos e cuidados de longa duração}

Pode-se inferir que a abordagem tradicional de gestão da atenção prestada ao paciente com DA com enfoque apenas na identificação e manejo dos sintomas apresentados são insuficientes. Pensando assim, existe a necessidade de médicos, gestores, profissionais da saúde, gerontólogos e políticos se concentrarem na prestação e implementação de serviços que favoreçam o planejamento da assistência de saúde das pessoas com DA e que possam manter a funcionalidade desses indivíduos por maior tempo possível, na tentativa de proporcionar uma assistência avaliada como sendo de excelência ${ }^{13}$.

O fator de maior custo para as pessoas com DA leve foi o do tempo do cuidador não remunerado, e para aqueles com a doença em estágio avançado foram os custos relativos à institucionalização $0^{13}$.

Pacientes com DA no estágio da doença moderada e grave são candidatos prováveis para a institucionalização. No entanto, as intervenções eficazes podem minimizar o avanço da incapacidade física, retardar a piora do déficit cognitivo e resultar no atraso de provável institucionalização. Tais prolongamentos aumentam as intervenções econômicas dos potenciais de custos a partir de U\$ 1.284 para U\$ 1.439 mensais em custos totais para cada mês de prolongamento. Em termos de gastos de serviços formais, a média de poupança mensal é de U\$ 2.029. Dessa forma, uma intervenção de seis meses poderia levar à economia de mais de U\$ 12 mil e, em um ano, poderia economizar mais de U\$24 mil por paciente. Melhorias ou retardamentos na progressão da doença também podem levar à redução de custos para cuidados informais. Contudo, as intervenções que atrasam a institucionalização inevitavelmente aumentaram os custos dos cuidados informais ${ }^{24}$.

Wolfs et al. ${ }^{26}$ ao pesquisarem 230 pacientes com demência, assistidos ambulatoriamente, na Holanda, por meio de estudo clínico randomizado, em uma amostra com 137 pacientes atendidos por uma equipe multidisciplinar, e um grupo controle de 93 pacientes que receberam cuidados usuais - concluíram que o uso do diagnóstico integrado e multidisciplinar mensurado pela função do bem-estar social a partir dos anos de vida ajustados pela qualidade (QALY) demonstrou resultados positivos relacionados ao custo/eficiência no diagnóstico e manejo de demência nos pacientes dementados ambulatoriais.

Nessa abordagem, a Alzheimer's Disease Internationa ${ }^{27}$ elaborou um modelo de ação formado por cinco fases visando o planejamento de serviços prestados aos portadores de demência, que pode ser utilizado em países com recursos limitados, com o intuito de melhorar a qualidade de vida desses pacientes e de seus cuidadores. Esse modelo está apresentado na Figura 1.

A fase inicial do modelo concentra-se na sensibilização e compreensão da DA pela sociedade e pelos gestores de saúde; na segunda fase identifica-se a necessidade da capacitação de familiares e profissionais da saúde em relação aos cuidados prestados aos pacientes com DA; a terceira alerta quanto à alimentação adequada, prática de exercícios físicos e interação social do paciente almejando a redução de riscos; e as últimas duas fases referem-se ao desenvolvimento dos serviços de

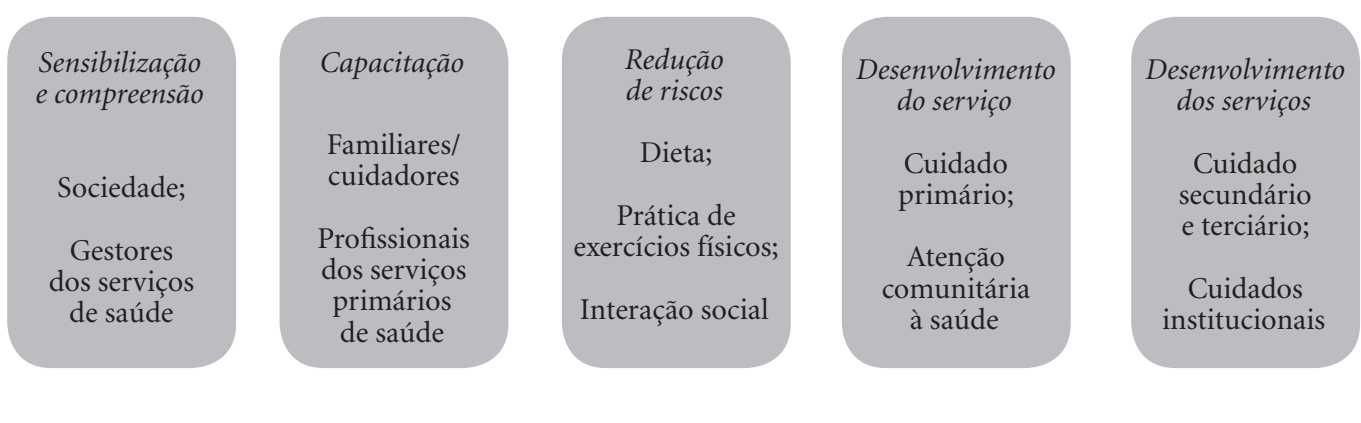

Figura 1. Abordagem gradual para o desenvolvimento de serviços a pessoas com demência. 
atenção primária e serviços de atenção secundária e terciária relacionados às instituições hospitalares e de longa permanência para idosos.

A partir desse modelo, espera-se que exista a prevenção ou o pelo menos o atraso da necessidade de serviços institucionais para os pacientes com DA, que são dispendiosos e que poucas famílias podem suportar financeira e emocionalmente. Acredita-se que em países onde o financiamento dos cuidados de saúde é severamente limitado torna-se essencial começar com iniciativas que tenham o máximo impacto junto de tantas pessoas quanto possível ${ }^{27}$.

Pesquisadores norte-americanos, também preocupados com a melhoria da assistência aos idosos com DA, idealizaram, nos EUA, o Comprehensive Alzheimer's Disease Centers (CADC), acreditando que esse serviço poderia reduzir pela metade os US\$ 150 milhões de custos anuais com a DA, que são estimados para a década de 2010-20. Assim, um investimento de US\$ 500 milhões, ou seja, menos de 1\% das despesas correntes anuais (US\$ 150 bilhões), com a DA dos EUA poderia levar à economia de mais de US\$ 75 bilhões em despesas de saúde em dez anos, e que está para além do efeito apenas dos CADC, visto que possibilita a criação de novos empregos e outros efeitos que poderão estimular a economia dos $\mathrm{EUA}^{28}$.

Nessa linha de pensamento, considera-se que a implantação de Centro Dia para Idosos na realidade brasileira mostra grandes perspectivas na melhoria da qualidade da assistência prestada ao familiar e paciente com DA, além da diminuição de custos sociais e públicos. Embora essa seja uma modalidade de atenção importante, tais iniciativas ainda são modestas no Brasil.

O Centro Dia para Idosos é um serviço de proteção social que oferece atendimento multiprofissional ao idoso, desenvolvendo atividades de promoção e proteção de saúde e a socialização de seus frequentadores. É destinado à permanência diurna de idosos com dependência parcial nas atividades de vida diária e que necessitam de assistência multiprofissional ${ }^{29}$.

Devido à escassez de estudos sobre esta modalidade de prestação de serviços no Brasil, o Departamento de Medicina, a partir das disciplinas de Geriatria e Gerontologia da UNIFESP-EPM, avaliou o impacto da assistência multiprofissional sobre a capacidade funcional de idosos atendidos em Centro Dia Geriátrico, fundado em $2005^{29}$. Apesar de alguns fatores limitantes, como a frequência e o número limitado de atendimentos no serviço, os idealizadores observaram que a assistência mostrou um impacto positivo sobre a capacidade funcional de idosos atendidos, revelando ser uma modalidade de assistência promissora na melhora funcional ${ }^{29}$.

A reabilitação funcional pode ser um dos pontos chave para o sucesso dessa modalidade assistencial. $\mathrm{O}$ aumento da independência relacionada às atividades básicas e instrumentais da vida diária em indivíduos com demência, por conta da prática de exercício, pode levar a uma melhor qualidade de vida, menos custos econômicos e menor atuação do cuidador ${ }^{30}$.

Os resultados desta análise sugerem que a dependência dos pacientes fornece uma contribuição significativa na explicação das variações nos custos da saúde na DA e que as pequenas mudanças na dependência estão associadas com grandes mudanças nos custos de atendimento. Assim, as intervenções que melhorem ou atrasem a dependência do paciente podem estar associadas à redução de $\operatorname{custos}^{30}$.

Nesta abordagem, Callahan et al..$^{31}$, ao testarem a eficácia de um modelo colaborativo de cuidados para melhorar a qualidade prestada a pacientes com DA, concluíram que tais cuidados resultaram em melhoria significativa na qualidade do atendimento e dos sintomas comportamentais e psicológicos da demência entre os pacientes de cuidados primários e seus cuidadores. Essas melhorias foram conseguidas sem aumentar significativamente o uso de antipsicóticos e sedativos hipnóticos.

O apoio aos familiares de idosos com DA também é efetivo para a redução de desfechos adversos associados à saúde do cuidador e, consequentemente, para os custos ocasionados pela exposição prolongada a situações de estresse e sobrecarga emocional. Segundo Zarit ${ }^{32}$, programas psicoeducativos e terapêuticos são eficazes para auxiliar familiares e cuidadores de idosos com DA, tendo como objetivos oferecer suporte emocional, trocar informações, construir conhecimentos, compartilhar dilemas e aprender maneiras de manejar estressores internos e externos relacionados ao cuidado.

Wilz e Fink-Heitz ${ }^{33}$, ao implementarem um programa de férias assistidas a cuidadoras de idosos com demência, observaram melhorias no estado emocional e físico das mesmas após três meses de intervenção, indicando que esta parece ser uma estratégia que pode ser implantada na rede de atenção a essa faixa etária. As férias foram planejadas por uma equipe de profissionais da saúde e tiveram duração de uma semana. Durante esse período as cuidadoras participaram de 
atividades lúdicas e de discussões temáticas sobre a doença e os cuidados oferecidos. Os pacientes com demência, por sua vez, foram assistidos pelas equipes de atenção do programa.

Na mesma direção, Eloniemi-Sulkava et al. ${ }^{34}$ descreveram que, ao estudarem um programa de atenção domiciliária oferecido a pacientes com DA, observaram a diminuição da probabilidade de institucionalização do idoso com demência nos dois anos de atendimento. Embora o estudo não tenha observado redução nos custos associados à atenção ao idoso, os autores constataram que a iniciativa reduziu a mobilização de serviços e recursos municipais. A priori, a iniciativa de atendimento domiciliário se mostrou efetiva por diminuir a institucionalização e minimizar os custos com a assistência.

Desse modo, torna-se importante o conhecimento e a redução da vulnerabilidade dos idosos assistidos no Centro Dia para Idosos, os programas de educação e a assistência domiciliária, pois estes dados poderão subsidiar as práticas de saúde e sociais, favorecendo a integração dos planos referentes aos indivíduos, aos programas e às políticas de saúde, de acordo com as necessidades da coletividade, por meio de trabalho com os grupos sociais e realização de diagnósticos sobre as condições da comunidade. Também se poderão redefinir os objetos de intervenção e realizar análises críticas das práticas de saúde e sociais, principalmente aquelas voltadas ao atendimento em Centro Dia para Idosos.

\section{Considerações finais}

O gerenciamento da atenção ao idoso portador de demência culmina na adoção de estratégias que implicam na participação da família, da comunidade e dos serviços de atenção formal, auxiliando no planejamento da atenção, na maximização do desempenho funcional e nos recursos que os cuidadores possuem para prover os cuidados. Estudos recentes indicam que evitar a perda funcional, a fragilidade e investir em programas de prevenção em saúde aumenta a qualidade de vida dos idosos, reduzindo os gastos em saúde ${ }^{35}$. Partindo da premissa que existe o aparecimento de um novo caso de demência no mundo a cada sete segundos, deverão existir políticas públicas que amparem tanto os idosos com DA quanto os seus familiares ${ }^{36}$.

Conforme Bloom et al. ${ }^{9}$, é possível que os custos com a DA nos próximos anos sejam mais elevados em virtude das poucas ações nas áreas de prevenção da dependência avançada e do declínio funcional. Caso não exista profilaxia ou tratamento eficaz da doença estaremos fadados a cuidar de idosos com potencial muito pequeno para convívio com a família e a comunidade.

Acredita-se que para atingir um resultado satisfatório na área de assistência prestada à pessoa com DA é fundamental o comprometimento de diferentes profissionais e setores da sociedade, mobilizando ações que deverão ser desenvolvidas a curto, médio e longo prazo, incluindo:

a) ampliação da rede de serviços que podem contribuir para maximizar o período em que os idosos com DA apresentarão maior independência, minimizando a velocidade de prejuízos associados à funcionalidade e a dependência para as AVD. Nesse sentido, o Centro Dia para Idosos é uma modalidade assistencial que merece ser estudada e implementada pelo poder público;

b) adoção de práticas de autocuidado do cuidador, sendo a família e o paciente assistidos por serviços formais de atenção e integrados à rede básica e complexa de saúde. Neste contexto, a rede de atenção básica de saúde pode se instrumentalizar propondo programas de atenção domiciliária e os serviços complexos à retaguarda necessária para que o idoso e seus familiares sejam assistidos por profissionais clínicos especializados;

c) otimização dos serviços já existentes, como Unidades Básicas de Saúde (UBS), Unidade de Referência à Saúde do Idoso (URSI), Centros Dia para Idosos e hospitais psicogeriátricos;

d) necessidade de convidar a família e a comunidade para participarem da gestão do atendimento ao idoso, melhorando a comunicação entre a família e os profissionais da atenção em saúde e atenção social;

e) efetivação de ações de educação dos profissionais em relação às demências e às possibilidades de cuidado, o que poderia maximizar os tipos de encaminhamentos e intervenção mobilizados, com enfoque, neste caso, para a redução de custos e para a maximização da independência e bem-estar do idoso e da família;

f) implementação de políticas públicas que favoreçam possibilidades de melhorias na atenção prestada aos idosos com DA e aos seus familiares;

g) ampliação de pesquisas que contribuam para as mudanças de paradigmas relacionados ao envelhecimento e velhice no Brasil.

Fazem-se necessárias mudanças emergentes relacionadas à gestão da atenção ao idoso com DA, tanto na área social quanto na da saúde, nor- 
teada pela efetivação das políticas públicas que versam sobre a prestação de apoio, assegurando as necessidades dos familiares e dos pacientes, propiciando, assim, a diminuição de custos relacionados à DA.

\section{Colaboradores}

BAO Gutierrez, HS Silva, C Guimarães e AC Campino participaram igualmente de todas as etapas de elaboração do artigo.

\section{Referências}

1. World Health Organization (WHO). Dementia: a public health priority. World Health Organization 2012. [acessado 2010 out 22]. Available from: http://whqlibdoc.who.int/publications/2012/9789241564458_eng. pdf

2. Winblad B. Socio-economic perspectives of dementia and costeffectiveness of treatment. $7^{\text {th }}$ International Geneva/Springfield. Symposium on Advances in Alzheimer Therapy, April 3-6, Geneva, Switzerland. 2002.

3. Hebert LE, Scherr PA, Bienias JL, Bennett DA, Evans DA. Alzheimer disease in the USA population: prevalence estimates using the 2000 census. Arch Neurol 2003; 60(8):1119-1122.

4. Small GW, Donohue JA, Brooks RL. An economic evaluation of donepezil in the treatment of Alzheimer's disease. Clin Ther 1998; 20(4):838-850.

5. Fratiglioni L, De Ronchi D, Aguero-Torres H. Worldwide prevalence and incidence of dementia. Drugs Aging 1999; 15(5):365-375.

6. Hebert LE, Scherr PA, Beckett LA, Albert MS, Pilgrim DM, Chown MJ, Funkenstein HH, Evans DA. Age-specific incidence of Alzheimer's disease in a community population. JAMA 1995; 273(17):1354-1359.

7. Burlá C, Camarano AA, Kanso S, Fernandes D, Nunes R. Panorama prospectivo das demências no Brasil: um enfoque demográfico. Cien Saude Colet 2013; 18(10):2949-2956

8. Wimo A, Winblada B, Linus JL. The worldwide societal costs of dementia: estimates for 2009. Alzheimers Dement 2010; 6(2):98-103,

9. Bloom BS, Pouvourville N, Straus WL. Cost of illness of Alzheimer's Disease: How useful are current estimates? Gerontologist 2003; 43(2):158-164.

10. Meek PD, McKeithan K, Schumock GT. Economic considerations in Alzheimer's disease. Pharmacotherapy 1998; 18(Pt. 2):68-73.

11. Brookmeyer R, Gray S, Kawas C. Projections of Alzheimer's disease in the United States and the public health impact of delaying disease onset. Am J Public Health 1998; 88():1337-1342.

12. McCallion H. Dementia Training. Mental Health Practice 2009; 12(7):8.

13. Hux MJ, O’Brien BJ, Iskedjian M, et al. Relation between severity of Alzheimer's disease and costs of caring. CMAJ 1998; 159(5):457-465. 
14. Schneider J, Murray J, Banerjee S, Mann A. EUROCARE: a cross-national study of co-resident spouse carers for people with Alzheimer's disease: I-Factors associated with carer burden. Int. J Geriatr Psychiatry 1999; 14(8):651-661.

15. Cuijpers P. Depressive disorders in caregivers of dementia patients: a systematic review. Aging Ment Health 2005; 9(4):325-330.

16. Lopes MA, Bottino CMC. Prevalência de demência em diversas regiões do mundo: análise dos estudos epidemiológicos de 1994 a 2000. Arq Neuropsiquiatr 2002; 60(1):61-69.

17. Nitrini R, Bottino CMC, Albala C, Custodio Capuñay NS, Ketzoian C, Llibre Rodriguez JJ, Maestre GE, Ramos-Cerqueira AT, Caramelli P. Prevalence of dementia in Latin America: a collaborative study of population -based cohorts. Int Psychogeriatr 2009; 21(4):622-630.

18. Scazufca M, Menezes PR, Vallada HP, Crepaldi AL, Pastor-Valero, Coutinho MS, Di RienzoV, Almeida P. High prevalence of dementia among older adults from poor socioeconomic backgrounds in São Paulo, Brazil. Int Psychogeriatr 2008; 20(2):394-405.

19. Veras P, Caldas CP, Dantas SB, Sancho LG, Sicsú B, Motta LB. Demented elderly people living at home in Rio de Janeiro, Brazil: Evaluation of expenditure care. Psychogeriatrics 2008; 8(2):88-95.

20. Karsch UM. Idosos dependentes: famílias e cuidadores. Cad Saude Publica 2003; 19(3):861-866.

21. Lebrão ML, Duarte YAO, organizadores. Sabe - Saúde, bem-estar e envelhecimento - O projeto Sabe no município de São Paulo: uma abordagem inicial. Brasília: Organização Pan-Americana da Saúde (OPAS); 2003.

22. Giacomin KC, Peixoto SV, Uchoa E, Lima-Costa MF. Estudo de base populacional dos fatores associados à incapacidade funcional entre idosos na Região Metropolitana de Belo Horizonte, Minas Gerais, Brasil. Cad Saude Publica 2008; 24(6):1260-1270.

23. Lima-Costa MF, Barreto SM, Giatti L. Condições de saúde, capacidade funcional, uso de serviços de saúde e gastos com medicamentos da população idosa brasileira: um estudo descritivo baseado na Pesquisa Nacional por Amostra de Domicílios. Cad Saude Publica 2003; 19(3):735-743.

24. Alzheimer's Society. Financial cost of dementia. [acessado 2010 fev 28]. Available at: http://www.alzheimers. org.uk/site/scripts/documents_info.php?document ID $=418$

25. Zhu C, Leibman C, McLaughlin T, Zbrozek AS, Scarmeas N, Albert M, Brandt J, Blacker D, Sano M, Stern Y. Patient dependence and longitudinal changes in costs of care in Alzheimer's Disease. Dement Geriatr Cogn Disord 2008; 26(5):416-423.

26. Wolfs CAG, Dirksen CD, Kessels A, Severens JL, Verhey FR. Economic evaluation of an integrated diagnostic approach for psychogeriatric patients. Arch Gen Psychiatry 2009; 66(3):313-323.
27. Alzheimer's Disease International. World Alzheimer Report. 2009. [acessado 2010 out 22]. Available at: http:// www.alz.co.uk/research/files/WorldAlzheimerReport -ExecutiveSummary.pdf

28. Trojanowski JQ, Arnold SE, Karlawish JH, Brunden K, Cary M, Davatzikos C, Detre J, Gaulton G, Grossman M, Hurtig H, Jedrziewski K, McCluskey L, Naylor M, Polsky D, Schellenberg GD, Siderowf A, Shaw LM, Van Deerlin V, Wang LS, Werner R, Xie SX, Lee VM. Design of comprehensive Alzheimer's disease centers to address unmetnational needs. Alzheimers Dement 2010; 6(2):150-155.

29. Franciulli, SE, Ricci NA, Lemos ND, Cordeiro RC, Gazzola JM. A modalidade de assistência Centro-Dia Geriátrico: efeitos funcionais em seis meses de acompanhamento multiprofissional. Cien Saude Colet 2007; 12(2):373-380.

30. Prigerson HG. Costs to society of family caregiving for patients with end-stage Alzheimer's disease. $N$ Engl J Med 2003; 349(20):1891-1892.

31. Callahan CM, Boustani MA, Unverzagt FW, Austrom MG, Damush TM, Perkins AJ, Fultz BA, Hui SL, Counsell SR, Hendrie HC. Effectiveness of collaborative care for older adults with Alzheimer disease in primary care: a randomized controlled trial. JAMA 2006; 295(18):2148-2157.

32. Zarit SH. Empirically supported treatment for family caregivers. In: Qualls SH, Zarit SH, editors. Aging families and caregiving. Hoboken, New Jersey: John Wiley \& Sons Inc; 2009. p. 131-154.

33. Wils G, Fink-Heitz M. Assisted vacations for men with dementia and their caregiving spouses: evaluation of health-related effects. Gerontol 2008; 48(1):115-120.

34. Eloniemi-Sulkava U, Saarenheimo M, Laakkonenm M-L, Pietilä M, Savikko N, Kautiainen H, Tilvis RS, Pitkälä KH. Family care as collaboration: effectiveness of a multicomponent support program for elderly couples with dementia. Randomized controlled intervention study. JAGS 2009; 57(12):2200-2208.

35. Caldas CP, Veras RP, Motta LB, Lima KC, Kisse CBS, Trocado CVM, Guerra ACLC. Rastreamento do risco de perda funcional: uma estratégia fundamental para a organização da Rede de Atenção ao Idoso. Cien Saude Colet 2013; 18(12):3495-506

36. The Alzheimer's Study Group Report. A National Alzheimer's Strategic Plan. The Report of the Alzheimer's Study Group. 2008. [acessado 2010 nov 30]. Available at: www.alz.org/news_and_events_16243.asp

Artigo apresentado em 15/03/2013

Aprovado em 05/04/2013

Versão final apresentada em 07/04/2013 